\title{
Fractal fluctuations in gaze speed visual search
}

\author{
Damian G. Stephen • Jason Anastas
}

Published online: 24 December 2010

(C) Psychonomic Society, Inc. 2010

\begin{abstract}
Visual search involves a subtle coordination of visual memory and lower-order perceptual mechanisms. Specifically, the fluctuations in gaze may provide support for visual search above and beyond what may be attributed to memory. Prior research indicates that gaze during search exhibits fractal fluctuations, which allow for a wide sampling of the field of view. Fractal fluctuations constitute a case of fast diffusion that may provide an advantage in exploration. We present reanalyses of eye-tracking data collected by Stephen and Mirman (Cognition, 115, 154-165, 2010) for single-feature and conjunction search tasks. Fluctuations in gaze during these search tasks were indeed fractal. Furthermore, the degree of fractality predicted decreases in reaction time on a trial-by-trial basis. We propose that fractality may play a key role in explaining the efficacy of perceptual exploration.
\end{abstract}

Keywords Visual search · Fractality · Exploration ·

Perception · Diffusion

Search is a fundamental problem for the behavioral sciences. Complex behaviors require complex resources,

D. G. Stephen $(\bowtie)$

Wyss Institute for Biologically Inspired Engineering,

Harvard University,

3 Blackfan Circle, Floor 5,

Boston, MA 02115, USA

e-mail: damian.stephen@wyss.harvard.edu

D. G. Stephen

Department of Psychiatry,

Children's Hospital Boston and Harvard Medical School,

Boston, MA, USA

\section{J. Anastas}

Department of Psychology, University of Connecticut,

Storrs, CT, USA and we live in environments cluttered with information, nutrients, and tools that serve diverse purposes. Complex behaviors depend on our ability to selectively sample the clutter. Depending on one's intention, not just any object will do. For example, finding a draft in a stack of papers will not help one start a car; finding car keys will not help one start revisions. In another example, foraging animals must distinguish between plants, poisonous and not, and between other animals, predators and prey, and they must know which to pursue and which to escape. Orienting toward the appropriate targets and away from unhelpful distractions or dangers is essential to intelligent, adaptive behavior.

\section{Visual search: coordination of memory with lower-order perceptual mechanisms}

Visual search is an intricate coordination of the visual perceptual system with optical information in the visual field. It involves locating target stimuli in spite of distracting stimuli. Some evidence indicates that visual perception in search relies on a serial integration of features (Treisman, 1996; Treisman \& Gelade, 1980). On another account, parallel processing may support serial integration in guiding search to likely targets (Wolfe, Cave, \& Franzel, 1989). Attentional shifts during visual processing appear to be supported by shortterm visual memory (Alvarez \& Cavanagh, 2004; Treisman, 1996) and by specific brain regions - for example, the dorsal frontoparietal region (Fairhall, Indovina, Driver, \& Macaluso, 2009) and the temporal-parietal junction (Mavritsaki, Allen, \& Humphreys, 2010).

The role of memory in shifts of attention during visual search has often found expression as the inhibition of return (IOR). IOR is the tendency for gaze to avoid previously viewed locations and to seek out locations that have not 
been viewed before (Posner \& Cohen, 1984; Posner, Rafal, Choate, \& Vaughn, 1985). Such a strategy relies on memory for what has been attended and maximizes the efficiency of new shifts of attention (Shore \& Klein, 2000). The evidence suggests that eye movements reflecting IOR support shifts of attention during visual search (Klein, 2000; Snyder \& Kingstone, 2007). Encompassing a pathway of control from memory to attention to eye movements, IOR represents an essential higher-order cognitive function driving search behaviors.

In addition to higher-order cognitive functions such as memory, there may be lower-order perceptual mechanisms that play a crucial role in visual search. A compelling example comes from research on visual search in participants with autism spectrum disorders (ASDs). ASDs compose a spectrum of social and communicative impairments (Kanner, 1943; Rutter, 1983). However, the relationship between ASDs and cognitive function has been controversial (Baron-Cohen, 1988; Happé, 1999; Hill \& Frith, 2003). Despite the evidence of some cognitive impairments in participants with ASDs, however, visual working memory in participants with ASDs is not different from that in age-matched typical participants (e.g., Ozonoff \& Strayer, 2001). Nonetheless, participants with ASDs perform visual search at significantly greater speeds than do typically developing (TD) participants (O'Riordan, 2004; O'Riordan, Plaisted, Driver, \& Baron-Cohen, 2001). O'Riordan and colleagues concluded that, in the absence of visual working memory impairments, there must be a lower-order perceptual mechanism at work that can provide significant advantages in search behaviors. Subsequent research suggested that there is no difference in number or spatial distribution of fixations during visual search by participants with ASDs and that the only difference from TD participants, at the coarse grain of fixations, is that participants with ASDs made shorter fixations (Joseph, Keehn, Connolly, Wolfe, \& Horowitz, 2009). As in IOR, visual search is best when eyes linger less and fluctuate more. However, the efficacy of visual working memory in participants with ASDs suggests that there may be more to evidence of IOR than simply higher-order cognitive functions supporting memory.

\section{Inhibition of return and hyperdiffusivity}

Perhaps some portion of IOR can be profitably understood in terms of physiological fluctuations. Recent research has made it unclear whether IOR can be helpfully distinguished from habituation, the tendency of physiological systems underpinning attention to fluctuate away from - rather than entrain to-previously attended stimuli (Dukewich, 2009; Robertson \& Johnson, 2008).
Previous research has shown that gaze fluctuations can be hyperdiffusive (Stephen, Mirman, Magnuson, \& Dixon, 2009). That is, gaze fluctuates faster and further than might be expected from ordinary mechanics. Under the central limit theorem (CLT), diffusion is a relationship between root-mean square (RMS) fluctuations (i.e., standard deviations $[S D \mathrm{~s}])$ of positions in a random walk and a power of time $t$ :

$S D(t) \sim t^{H}$,

where the scaling exponent $H$ determines speed of diffusion. We wish to emphasize that Eq. 1 does not hold for the $S D$ of a sample of individual displacements but, rather, for the random-walk trajectory integrated from the individual displacements. For ordinary diffusion, $S D$ grows as a square root of $t$-that is, $H=.5$. For hyperdiffusion under CLT, $S D$ grows faster, according to $H>.5$. Hyperdiffusion manifests as fractal fluctuations when $.5<H \leq 1$. For fractal fluctuations, Eq. 1 describes a power law, whose self-similar form entails similarly fast fluctuations across multiple scales of measurement (Scafetta \& Grigolini, 2002; Shlesinger, Zaslavsky, \& Klafter, 1993). This power law on the time domain entails that fractal fluctuations under CLT bear long-range temporal correlations. Aks, Zelinsky, and Sprott (2002) have suggested that fractal fluctuations in relative gaze positions may reflect the role of memory supporting IOR in visual search as temporal correlations in the physiology of the visual system (Baddeley et al., 1997; Billock, de Guzman, \& Kelso, 2001; Lowen, Ozaki, Kaplan, Saleh, \& Teich, 2001). This article will investigate whether fractal fluctuations facilitate visual search and, specifically, whether changes in fractality (i.e., changes in $H$ within the fractal range) predict changes in visual search reaction time (RT).

We do not intend to propose that the phenomenon of IOR should be fully accounted for by hyperdiffusion. IOR is a memory-driven mechanism proposed to promote fluctuations of gaze in the service of visual search, but the work of O'Riordan and colleagues (O'Riordan, 2004; O'Riordan et al., 2001) suggests that there may be differences in visual search not attributable to memory-driven mechanisms. Hence, the fluctuations in gaze serving visual search need not be exhaustively attributable to IOR. Rather than begin from the assumption that IOR accounts for all fluctuations in gaze, we sought only to investigate what relevance the measured diffusivity of fluctuations, in general, might have for visual search.

Distinction from alternate applications of diffusion in cognitive sciences We wish to distinguish this application of diffusion from that found in work by Ratcliff and colleagues (e.g., Ratcliff, 1978; Wagenmakers, Ratcliff, 
Gomez, \& McKoon, 2008). The work by Ratcliff and colleagues models the distribution of RT for binary responses as a function of internal, mental diffusion processes. This approach generally involves inferring diffusive processes within the cognitive architecture that gives rise to a measured distribution of RT and, subsequently, simulating RT distributions from models of such inferred diffusive processes. The present application involves estimating diffusivity from RMS fluctuations from overt, measured perceptual-motor time series to predicting changes in RT. The outcome measure in the present work is RT in a cognitive task, but otherwise, the present approach does not imply a diffusive process within the cognitive architecture. Instead, the article investigates the relationship between measured diffusivity and measured RT.

\section{Fractal fluctuations in gaze during visual search}

Hypothesis 1 Previous work has documented fractal fluctuations in gaze. Typical fluctuation measures include the distances between gaze positions in either one dimension $(\Delta x$ or $\Delta y)$ or two dimensions $\left[\left(x^{2}+y^{2}\right)^{1 / 2}\right]$ and the angular changes with respect to the horizon between gaze positions (i.e., the arctangent of $\Delta y / \Delta x$; Aks, 2005). In a visual search task requiring participants to identify an upright " $\mathrm{T}$ " amid rotated "Ts," both one-dimensional gaze distance measures exhibited fractal fluctuations, and the distance measure exhibited fractal ranges over longer time scales (Aks et al., 2002). Given static images of interlocking gears, for participants determining the turning direction of the final gear, angular change of gaze exhibited fractal fluctuations (Stephen, Boncoddo, Magnuson, \& Dixon, 2009). Our first prediction (Hypothesis 1) is that angular change of gaze exhibits fractal fluctuations during visual search.

A note on gaze measures and fractal analysis Distance between individual gaze positions (i.e., gaze steps) may be unsuitable for analysis by finite variance scaling methods (FVSMs) such as detrended fluctuation analysis (DFA; Stephen, Mirman, et al., 2009). Contributing to heavy tails in the aggregate distribution of gaze steps, saccades will bias the $S D$ of the random-walk trajectory integrated from the individual gaze steps. Similar heavy tails can be found in the gaze steps during single-feature and conjunction search (Stephen \& Mirman, 2010). Despite the incompatibility of standard fractal measures with fluctuations in gaze position, we investigate the temporal fractality of fluctuations in gaze orientation. Measures of fluctuations in gaze orientation will not be contaminated by saccades in the same way as fluctuations in gaze position. Although not often used in the literature, the arctangent measure provides a measure of fluctuations in orientation ideal for examining temporal structure using FVSMs. The arctangent measure provides a measure of angular change with respect to the horizon. The bounds on the arctangent function guarantee that the aggregate distribution of these fluctuations in gaze orientation will not suffer from heavy tails. The arctangent measure does not preserve all of the information about directionality, but it allows a mathematical compromise between directionality and evidence of temporal structure. ${ }^{1}$

Although a distance measure would be a more conceptually direct anchor for the notion that exploratory behaviors diffuse quickly through a space, the angular measure should work as well. A crucial factor guaranteeing the fast growth of $S D$ in hyperdiffusion is temporal correlation (Reynolds, 2010). Temporal correlations in gaze orientation would entail that gaze moves persistently - that is, describing sustained movement in similar directions across multiple samples (e.g., as in saccades). Absence of temporal correlations in angular change across samples would leave gaze more circuitous and, hence, slower to reach new regions of the visual display. The $S D$ increasing quickly is not a random-walk trajectory in the twodimensional Cartesian space of the screen; instead, it is a random-walk trajectory along a one-dimensional angular space of orientations with respect to the horizon.

Hypothesis 2 We also pursue Aks et al.'s (2002) notion that fractal fluctuations in gaze may support visual search. As was noted above, we do not propose that hyperdiffusivity or fractality accounts for the memory-driven (i.e., cognitive) mechanism of IOR. We seek to test the role of temporal fractality insofar as it may index key aspects of the lower-order perceptual (i.e., noncognitive) mechanisms proposed to influence visual search (e.g., O'Riordan, 2004). Note that this work does not seek to attribute fractality to a higher-order cognitive function but only to investigate a

\footnotetext{
${ }^{1}$ Zeros indicate samples when changes in $x$ or $y$ equaled 0 , leaving the arctangent function either undefined or zero. Otherwise, the range of the arctangent function is constrained to the range $-\pi / 2$ to $\pi / 2$, but there is no simple way to leave the range of angular changes unconstrained that would not also misrepresent evidence of temporal correlations. If the angles were all unsigned, it would be difficult to express correlations among similar angular changes (e.g., angular changes of $5^{\circ}$ and $355^{\circ}$ entail similar changes in orientation, but the magnitudes of these angles in sequence would not support a statistical measure of temporal correlation). However, if the signed angular changes may be used to partially encode direction, leaving the absolute value of the angular changes too large leaves the same problem (e.g., angular changes of $-175^{\circ}$ and $175^{\circ}$ pose largely the same problem as $5^{\circ}$ and $355^{\circ}$ in the earlier example). In short, the arctangent is a rough measure of angular change but not a perfect one. However, the challenge of encoding angular change in gaze orientation is by no means trivial.
} 
possible relationship between cognitive performance (i.e., RT in visual search) and fractality of gaze.

Our second hypothesis anticipates a negative relationship between the temporal fractality of angular change and RT in visual search. The similarity in spatial distribution covered by gaze in ASD and TD groups (see Joseph et al., 2009) does not recommend the prediction that fractal fluctuations in gaze distance measures should predict RT in visual search. However, perhaps the shorter fixations of the ASD group arose from fractal fluctuations in gaze orientation (i.e., angular change). Furthermore, fractal fluctuations in angular change of gaze have precedence for predicting individual differences in visual-cognitive performance. In the gear task described above, trial-by-trial changes in fractality of angular change of gaze predicted individual differences in how soon (over trials) participants discover the parity rule relating even-/oddness of gear number and final turning direction (Stephen, Boncoddo, et al., 2009). It may be that fractal fluctuations in angular change will similarly predict how soon (within trials) participants can decide whether or not a target stimulus is present on the screen. Our second prediction is that fractal fluctuations in angular change of gaze will predict individual differences in visual search RT. Because the scaling exponent $H$ indicates speed of diffusion (i.e., Eq. 1, growth of $S D$ ), greater $H$ will reflect faster diffusion of gaze and will, consequently, lead to faster visual search (i.e., lower RT). Hence, Hypothesis 2 is that greater fractality in angular change of gaze will lead to lower RT.

We will reanalyze Stephen and Mirman's (2010) eyetracking work in visual cognition. Stephen and Mirman examined gaze fluctuations during single-feature and conjunction searches, as well as during a visual world paradigm (VWP; Tanenhaus, Spivey-Knowlton, Eberhard, \& Sedivy, 1995). They replicated the interaction between set size and conjunction (Treisman \& Gelade, 1980). They examined gaze fluctuations only in aggregate - that is, analyzing probability distribution functions by task, collapsed over trials. We will reanalyze gaze data from the single-feature and conjunction searches. The test of Hypothesis 1 will involve first testing for long-range memory and next estimating trial-by-trial $H$ through fractal analysis of each trial's time series of angular change. In order to test Hypothesis 2, we will test for an effect of $H$ on RT beyond the positive effect of set size in the conjunction search.

\section{Method}

Six participants with normal to corrected-to-normal vision completed two visual search tasks. In the original data collection, participants completed three visual-cognitive tasks in counterbalanced order: single-feature and conjunction searches as well as a VWP task. We omit the VWP task in this reanalysis, noting that analyses have tested block as a predictor varying both from 1 to 3 and from 1 to 2 . The single-feature task required indicating whether or not the letter $O$ was present among distractor letters $N$ and $X$. The conjunction task required indicating whether or not a green letter $N$ was present among distractors including green $X \mathrm{~s}$ and brown $N$ s. Participants completed the visual search tasks in blocks of 24 trials ( 6 trials for each set size of 1,5 , 15, and 30 letters). All participants provided accurate responses at least $87.5 \%$ of the time. ${ }^{2}$ Logistic regressions of trial-by-trial accuracy demonstrated no significant effect of speed and, hence, consistent with O'Riordan's (2004) results from participants with ASD, no speed-accuracy trade-off.

\section{Data analysis}

Testing the two hypotheses will require a variety of analyses. Testing Hypothesis 1-namely, that fluctuations in angular change of gaze are fractal-will primarily involve using DFA (Peng et al., 1994) to estimate trial-by-trial scaling exponents $H$. However, because DFA can generate a false diagnosis of long-range temporal correlations (Maraun, Rust, \& Timmer, 2004; Wagenmakers, Farrell, \& Ratcliff, 2004, 2005), we should also submit the data to two other analyses for corroboration of long-range temporal correlations: autoregressive fractionally integrated moving average (ARFIMA; Granger \& Joyeux, 1980) modeling and dispersion analysis (Bassingthwaighte, Liebovitch, \& West, 1994; Holden, 2005). In the presence of converging evidence for long-range temporal correlations, DFA yields relatively more reliable estimates of scaling exponents $H$ (Oswiecimka, Kwapien, \& Drozdz, 2006; Peng et al., 1994; Torre, Delignières, \& Lemoine, 2007). Hence, the DFA estimates of $H$ will subsequently serve for the test of Hypothesis 2. To test Hypothesis 2-namely, that greater fractality will lead to lower RT - we will use growth curve modeling (GCM; Singer \& Willett, 2003). GCM is ideal for testing the effect of time-varying predictors, and we will use it to test whether changes in $H$ predict changes in RT. We describe the above DFA and GCM in the subsections that follow, and because ARFIMA modeling and dispersion analysis serve more auxiliary roles in the present work, we leave description of these methods to the Appendix.

Detrended fluctuation analysis DFA first integrates an $N$ length increment time series $x(t)$, producing a cumulative-

\footnotetext{
${ }^{2}$ For further details of original data collection, see Stephen and Mirman (2010).
} 
sum time series $y(t)$ where each $i$ th value is the sum of the $i$ th value of $x$ and all previous values of $x$ :

$y(t)=\sum_{i=1}^{N} x(i)-\overline{x(t)}$

where $\overline{x(t)}$ is the mean of $x(t)$. As was noted above, evidence of hyperdiffusion will be a fast growth of $S D$ not in the individual displacements (i.e., $x$ ) but in the random-walk trajectory (i.e., $y$ ) integrated from the individual displacements. Linear regressions $y_{n}(t)$ detrend nonoverlapping $n$ length windows of $y(t)$. Fluctuation $F(n)$ is calculated as the average RMS error of these regressions for each $n$ :

$F(n)=\sqrt{(1 / N) \sum\left[y(t)-y_{n}(t)^{2}\right]}$

for $n<N / 4$. Similar to $S D$ in Eq. $1, F(n)$ increases as

$F(n) \sim n^{H}$.

That is, DFA expands the basic formalism of Eq. 1 to a more general case of fluctuations with local trends. Whereas the mean is the best summary estimate of a trendless random-walk trajectory, a regressed line is the best summary estimate of position in trended fluctuation. So, whereas Eq. 1 describes $S D$ around a mean, Eq. 4 describes RMS around a regressed line. Fractal time series generally exhibit scaling exponents $H$ in the range $.5<H \leq 1$, with stronger fractality yielding an $H$ closer to 1 (e.g., Kiefer, Riley, Shockley, Villard, \& Van Orden, 2009). Logarithmic scaling of Eq. 4 yields

$\log F(n) \sim H \log n$.

The slope of $F(n)$ in double-log plots is taken to estimate $H$.

Growth curve modeling GCM is a maximum-likelihood (ML), hierarchical linear regression technique well suited to studying time-varying dynamics in visual cognition (Mirman, Dixon, \& Magnuson, 2008; Stephen, Boncoddo, et al., 2009). As in ordinary least-squares (OLS) regression, a time-varying dependent measure is modeled as the weighted sum of main effects and interactions. Whereas OLS estimation assumes equal variance across measurements (i.e., across time and participants), ML estimation permits random effects to capture individual differences over time, allowing GCM to estimate fixed effects more judiciously. GCM serves the same purpose as OLS methods typically would - namely, testing linearly separable effects of predictors on a dependent measure. It merely does so in a way that makes fewer assumptions about the structure of the dependent measure across time and across participants and that allows more flexibility in modeling effects for predictors varying over time.
A major difference between OLS regression and MLestimated GCMs lies in the use of $R^{2}$ as a goodness-of-fit statistic. $R^{2}$ expresses the proportion of variance explained by an OLS model, and OLS estimation evaluates added predictors in terms of increases in $R^{2}$. The very notion of finite variability of which a proportion might be "explained" by a model is founded deeply in the assumptions of OLS estimation. ML estimation for continuous dependent measures does not respect an absolute goodness-of-fit statistic, and so there is no sensible description of proportion of variance explained (Heinrich, 2003; Lyons, 2008). What ML does allow is a comparison of logarithmic (i.e., "log") likelihood before and after inclusion of a predictor. ML estimation evaluates added predictors as reductions in $-2 \log$ likelihood (-2LL). Change in -2LL may be tested as a chi-square statistic with as many degrees of freedom as added predictors (Singer \& Willett, 2003). For instance, if $m$ predictors are added to the model, and if $-2 \mathrm{LL}$ consequently drops by $w$ [i.e., $\left.w=-2 L L_{\text {Old }}-\left(-2 L L_{\text {New }}\right)\right]$, the improvement in model fit is treated as significant if the area under the chi-square curve for $m$ degrees of freedom from 0 to $w$ is greater than $95 \%$ of the total area under the curve.

\section{Results}

Testing Hypothesis 1: fractality of fluctuations in gaze orientation

Eye tracking during each trial provided one gaze time series, sampled at $500 \mathrm{~Hz}$, in two screen dimensions $(x, y)$. Six participants completed 24 trials in each of two blocks of visual search tasks, generating a total of 288 (i.e., $6 \times 2 \times$ 24) time series. Response included, on average, $1,686.84$ ( $S E=26.58)$ gaze positions; hence, RT was, on average, $3.37 \mathrm{~s}(S E=0.05)$. For each consecutive pair of gaze positions, angular change equaled the arctangent of $\Delta y / \Delta x$. Figure 1 shows an example angular-change time series.

We submitted all angular-change time series, as well as shuffled versions of each, to DFA. Since Aks and colleagues found crossovers (i.e., abrupt transitions) from temporal correlations beyond the fractal range in the high frequency (i.e., to temporal correlations in the fractal range; Aks, 2005; Aks et al., 2002), we excluded windows of size $n<7$ from the linear fit of the fluctuation function for estimating the scaling exponents $H$. Fractally fluctuating time series exhibit $H>.5$ in original sequence, but not when shuffled (Peng et al., 1994). The scaling exponents $H$ estimated for original angular-change time series $(M=.627$, $S E=.004)$ exceeded those estimated for the shuffled versions $(M=.478, S E=.003)$, paired-samples $t(287)=$ $25.98 p<.0001$. Figure 2 shows fluctuation functions for 


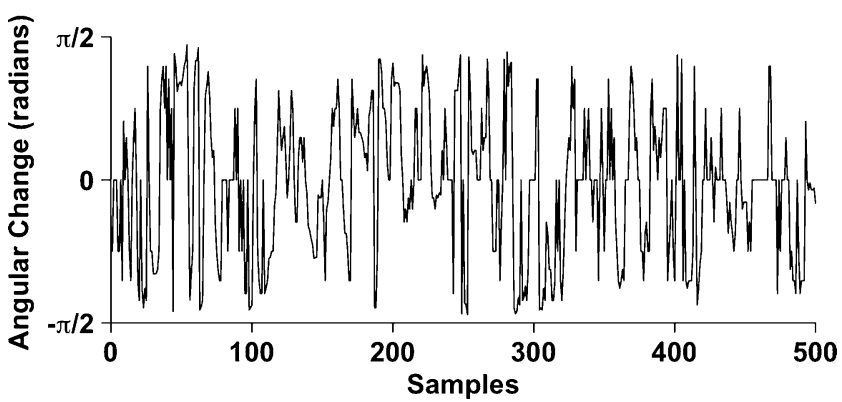

Fig. 1 Subset of 500 consecutive values from an example angularchange time series

an example time series in original and shuffled form. ${ }^{3}$ Should the further analyses provide converging evidence of long-range temporal correlations, this significant difference between the scaling exponents $H$ for the original time series and those for the shuffled versions would be evidence in favor of Hypothesis 1.

Additional tests for long-range temporal correlations: ARFIMA and dispersion analysis ARFIMA modeling of the angular-change time series performed better than ARMA modeling, suggesting the presence of long-range temporal correlations. ${ }^{3}$ Summed weights for ARFIMA models were higher than those for ARMA models according to the Akaike information criterion (AIC), paired-sample $t(287)=6.66, p<.001$, and according to the Bayesian information criterion (BIC), paired-sample $t(287)=4.00$, $p<.001$. The estimated value of $d(M=.072, S E=.002)$ from the best-fitting ARFIMA models according to AIC weights was significantly greater than zero for $89.58 \%$ of all 288 trials. The estimated value of $d(M=.047, S E=.004)$ from the best-fitting ARFIMA models according to the BIC was significantly greater than zero for $62.85 \%$ of all 288 trials. Both proportions were significantly higher than chance, $\chi^{2}(1)=180.50$ and $18.43, p s<.001$, according to the AIC and BIC, respectively. The theoretical relationship between the scaling exponent $H$ and the ARFIMA parameter $d$ is

$H=d+.5$,

and this relationship would yield mean values of $H=.572$ and .547 according to the AIC and BIC, respectively. The AIC estimate is close to the estimate of $H$ from DFA, but the BIC estimate is more conservative. In both cases, these parameters $d$ according to the AIC and BIC correlated with $H, r \mathrm{~s}(286)=.57$ and $.54, p \mathrm{~s}<.0001$, respectively.

\footnotetext{
${ }^{3}$ Preliminary to ARMA and ARFIMA modeling, the augmented Dickey-Fuller test for detecting nonstationarity (i.e., "unit roots"; Banerjee, Dolado, Galbraith, \& Hendry, 1993) rejected the hypothesis of nonstationarity for all 288 angular-change time series, $p<.05$.
}

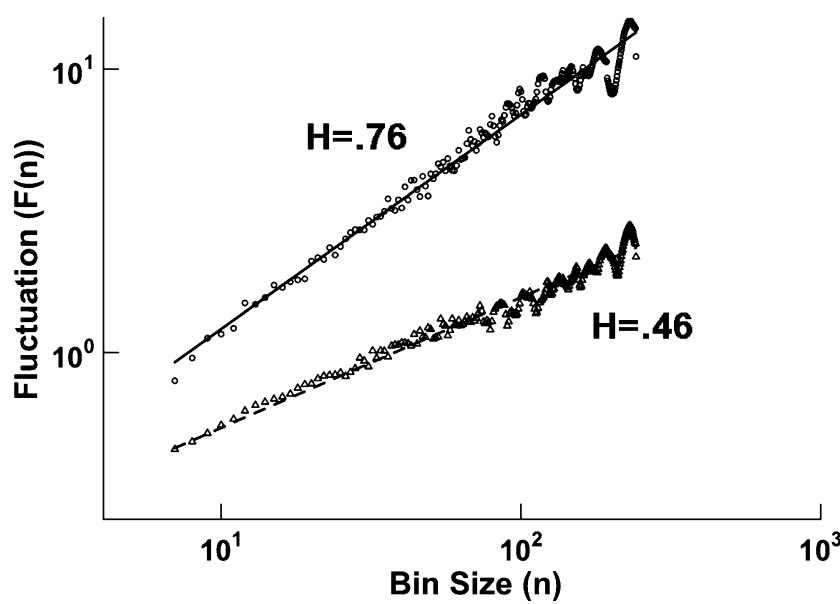

Fig. 2 Fluctuation functions for an example angular-change time series in original sequence and for the same time series in shuffled sequence

All angular-change time series were submitted to dispersion analysis. Dispersion analysis returned estimates of fractal dimension $(F D)$ consistent with temporal fractality and, hence, with long-range temporal correlations $(M=$ $1.412, S E=0.005)$. The theoretical relationship between $F D$ and $H$ is:

$H=2-F D$,

and this relationship would yield a mean value of $H=.588$, close to the observed $H$ estimated from DFA. FD correlated with $H, r \mathrm{~s}(286)=-.29, p<.0001$. Estimates of $F D$ for shuffled versions were consistent with additive white noise and, hence, with the absence of long-range temporal correlations $(M=1.546, S E=0.005)$.

Testing Hypothesis 2: effect of fractality on reaction time

GCMs replicated the effect of set size on RT during conjunction search (e.g., Treisman \& Gelade, 1980) and tested whether fractal gaze-orientation fluctuations reduces RT. Predictors controlling for practice or fatigue included trial (varying from 1 to 24 within block) and block (varying from 1 to 2$){ }^{4}$ Substantive predictors included task $(0=$ single-feature, 1 = conjunction), set size (varying among 1 , 5, 15, and 30 randomly across trials), and $H$ (from DFA).

Model 1 tested two interactions, trial $\times$ block and task $\times$ set size and constituent main effects (Table 1). It is important to note that no interaction can be tested judiciously without including the constituent main effects.

\footnotetext{
${ }^{4}$ Alternate models include block recoded to indicate block number across all three tasks in the original data collection. Besides marginally significant negative effects for this three-level block predictor, other predictors' effects did not change.
} 
Table 1 Coefficients from Model 1

\begin{tabular}{lllll}
\hline Predictor & $B$ & $S E$ & $t$ & $p$ \\
\hline Intercept & $3,540.17$ & 667.26 & 5.31 & $<.0001$ \\
Trial & -24.14 & 28.00 & -0.86 & .39 \\
Block & -406.35 & 348.35 & -1.17 & .27 \\
Task & 292.72 & 353.56 & 0.83 & .43 \\
Set size & 6.12 & 3.99 & 1.53 & .13 \\
Trial $\times$ order & 21.81 & 17.72 & 1.23 & .22 \\
Task $\times$ set size & 17.51 & 5.61 & 3.12 & $<.01$ \\
\hline
\end{tabular}

The only significant effect was the positive task $\times$ set size interaction $(B=17.51, S E=5.61, p<.01)$, indicating that RT increases with set size during conjunction search (task $=$ 1). Model 2 added the predictor $H$ to effects from Model 1 (Table 2), significantly improving prediction of RT, $\chi^{2}(1)=$ $6.57, p<.05$. The negative main effect for $H(B=-1357.91$, $S E=519.31, p<.01)$ indicates that RT decreased as $H$ increased. That is, the negative coefficient (i.e., $B$ weight) that Model 2 yields for $H$ indicates that increases in $H$ will predict decreases in the dependent measure-namely, $\mathrm{RT}^{5}$

\section{Discussion}

We made two hypotheses regarding fluctuations in the angular change of gaze. Hypothesis 1 was that these fluctuations might be fractal, meaning that their $S D$ would grow as a function of time- that is, $t^{H}$ (Eqs. 1 and 4), where $.5<H \leq 1$. Hypothesis 2 was that greater fractality, indexed by scaling exponent $H$, would predict lower RT beyond the effects of set size in a conjunction task. The results confirmed these hypotheses. DFA, ARFIMA modeling, and dispersion analysis provided converging evidence of the long-range temporal correlations indicative of fractality, thereby supporting Hypothesis 1. Drawing on the estimates of scaling exponents $H$ from DFA, GCM demonstrated the effect of fractality on RT in visual search. Specifically, the results showed that fractality of fluctuations in angular change had an effect on RT above and beyond - and in a direction opposing - the effect of set size in conjunction search. The latter result supported Hypothesis 2.

\footnotetext{
${ }^{5}$ Alternate estimates of temporal correlations exhibited converging evidence of similar effects on RT in alternate models building upon the foundation of Model 1. First, estimates of $H$ from a linear fit over a more conservative range of the fluctuation function (i.e., with upper bound $N / 10$, rather than the more conventional $N / 4$; e.g., Hu, Ivanov, Chen, Carpena, \& Stanley, 2001) also had a significant negative effect on RT. Second, the FD from dispersion analysis (which varies indirectly with $H$ ) had a marginally significant positive (i.e., in the opposite direction to $H$ ) effect on RT.
}

Fractality for gaze promotes visual search

The fractality of gaze influences perceptual exploration. Fractality of fluctuations of gaze entails that visual exploration samples the visual field with fluctuations that grow similarly over multiple time scales (note that similarity will generally be statistical, and not necessarily morphological). That is, visual exploration may be similarly complex over a variety of time scales. Gaze may never cease to fluctuate and, consequently, to sample the available visual information. As simulations of animal foraging have shown, fractal fluctuations ensure optimally thorough search behaviors (Bartumeus, Catalan, Fulco, Lyra, \& Viswanathan, 2002). Fractality, then, constitutes a departure of fluctuations from ordinary diffusion; this departure provides the visual system with important leverage for search.

These results may prove interesting because they highlight the role of fluctuations not traditionally credited with much causal force. It is typical in the cognitive sciences to attribute changes in processing time (i.e., RT) to contributions from higher-order cognitive faculties, such as visuospatial working memory, or to specialized brain structures. It has long been recognized that the subtle movements of the eye can be crucial for maintaining visual perception of an image (Barlow, 1963; Coppola \& Purves, 1996; Ditchburn \& Ginsborg, 1952; Helmholtz, 1962; Pritchard, Heron, \& Hebb, 1960; Riggs \& Ratliff, 1952; Riggs, Ratliff, Cornsweet, \& Cornsweet, 1953). However, the role of fluctuations has not so often been shown to influence performance usually attributed to the cognitive level of processing. In fact, there has been skepticism that eye movements can have implications even for more complex varieties of visual perception (e.g., visual perception of three-dimensional shape; Miller \& Festinger, 1977; Pizlo, 2008). However, we suggest that ocular fluctuations may not only support the passive experience of images, but may also support the efficiency with which the cognitive system engages in an intentional action with respect to an image. These results support the notion that cognitive function does not simply unfold through the activity of

Table 2 Coefficients from Model 2

\begin{tabular}{lllll}
\hline Predictor & $B$ & $S E$ & $t$ & $p$ \\
\hline Intercept & $4,392.41$ & 689.23 & 6.37 & $<.0001$ \\
Trial & -22.69 & 27.70 & -0.84 & .40 \\
Block & -382.93 & 323.00 & -1.21 & .26 \\
Task & 296.73 & 319.97 & 0.93 & .38 \\
Set size & 5.01 & 3.98 & 1.26 & .21 \\
$H$ & $-1,357.91$ & 519.31 & -2.61 & $<.01$ \\
Trial $\times$ order & 19.83 & 17.09 & 1.16 & .25 \\
Task $\times$ set size & 16.75 & 5.58 & 3.00 & $<.01$ \\
\hline
\end{tabular}


complex mental and neurological structures but is also deeply rooted in the lower-order perceptual-motor variability (e.g., O’Riordan, 2004; Stephen \& Mirman, 2010).

The apparent randomness of perceptual-motor "jitter" belies a deeper structure. The results reported here constitute further evidence of the strange kinetics of nonlinear energy flow (e.g., Shlesinger et al., 1993) in perceptual and cognitive tasks (e.g., Stephen, Mirman, et al., 2009). These findings emphasize the role of energy flow in cognitive processing (e.g., Friston, 2009; Kugler \& Turvey, 1987; Thelen \& Smith, 1994). Evidence of strange kinetics suggests that models of cognitive performance should not be built on strictly Newtonian terms of linear relationships among aggregate components; fluctuations at relatively small scales are not necessarily canceled out in aggregate behaviors but may have implications for events at relatively larger scales (Stephen, Boncoddo, et al., 2009). The long-range temporal correlations characterizing physiological fluctuations may be evidence of self-organizing processes supporting flexible, fluid cognitive performance (e.g., Kello, Beltz, Holden, \& Van Orden, 2007). Search behaviors may consequently grow naturally from the rich substrate of other fractally structured physiological events distributed throughout the nervous system (Werner, 2010; West, 2006). Furthermore, whatever the underlying process giving rise to the temporal correlations, the present work demonstrates that perceptual-motor fluctuations have tangible consequences for cognitive function.

Although we have proposed that fractality in visual search reflects an important aspect of lower-order perceptual mechanisms, it may still be tempting to conjecture as to the independent contribution of memory. For instance, the nonfractal design of visual search displays suggests that fractal visual search patterns reflect storage in memory of past experience with inspecting the fractal structure of natural scenes. However, the presence of long-range temporal correlation begins to blur the distinction between what may need to be stored in memory and what may not. Storage in memory may be absolutely necessary for a system whose variability in response to current stimuli carries no trace of variability in response to previous stimuli. However, temporal correlations in lower-order perceptual mechanism suggest that effects of a given stimulus are not simply wiped clean from the perceptual system following the removal of that stimulus; these effects may extend through time to influence the effect of later stimuli.

Furthermore, recent research on memory suggests that strength of recall bears a power law structure over time (Anderson \& Schooler, 1991; Brown, Neath, \& Chater, 2007; Rhodes \& Turvey, 2007). This finding is analytically different from the power law change in an $S D$ reported here, but it nonetheless implies fractal structure in the retention and retrieval of stored items in memory. If the visual search patterns are stored in memory, they may also be forgotten at a power law rate of time, and the availability of stored search patterns may thus be understood by further modeling of power law relationships. Thus, the actual relationship between memory and fractality may be rather complex and may demand a good deal more consideration and analysis than the scope of the present work allows. In any event, all we suggest is that the trial-by-trial changes in fractality may be predictive of efficiency of exploration as measured by RT.

Degree of fractality predicts individual differences in use of visual information

The scaling exponent $H$ expressing the strength of the power law relationship between fluctuations and time scale indexes the departure from ordinary diffusion. The present evidence of fractal fluctuations in angular change of gaze during search is consonant with existing research on gaze during visual search (Aks et al., 2002). However, it goes one step further, demonstrating that trial-by-trial scaling exponents for fluctuations in gaze predict trial-by-trial individual differences in visual exploration. Overall effects of set size in conjunction search produce differences in search in the aggregate (e.g., Treisman \& Gelade, 1980), but now we find that indexing changes in the diffusivity of eye movements predicts further differences in search. This finding resembles a comparable finding in haptic perception. When participants were asked to judge an unseen object's length after haptically exploring it through wielding, fractal fluctuations in wielding promoted accuracy, and the trial-bytrial scaling exponent predicted individual differences in the use of the unseen object's inertial moments for length judgments (Stephen, Arzamarski, \& Michaels, 2010). The present finding suggests that the role of fractality in perceptual exploration may hold not simply for the modality of haptics, but also for that of vision.

The aim of this article is to point to the powerful role that diffusion could play in understanding perceptual exploration. It has been proposed that fractality and selfsimilarity across multiple scales may represent a general framework in which perception, action, and cognition might be understood (Chater \& Brown, 1999; Van Orden, Holden, \& Turvey, 2003). These related notions should be understood as representing a subset of phenomena more generally known as diffusion (Stephen, Mirman, et al., 2009). We have attempted to present diffusion in terms of overt, measured perceptual-motor time series during search behaviors. The growth of fluctuations in gaze orientation allows the visual system to search a two-dimensional display. The speed of this growth - that is, the speed of gaze diffusion - is inversely related to the time needed for search. Fractality of fluctuations in gaze promotes visual search. Visual search represents a general concern for perceptual and cognitive tasks at large: detecting appropriate 
information in the task environment as efficiently as possible. Fractality in exploratory fluctuations may similarly be understood more generally as the fast diffusion of perceptual systems through a task environment. The quicker the diffusion and, hence, the more thorough the search, the more efficient the cognitive system may be. Exploring the diffusive properties of perceptual systems may thus be of utmost utility to cognitive science.

Acknowledgments The authors extend their gratitude to Daniel Mirman and James A. Dixon for their help in developing the work in this article.

\section{Appendix}

\section{ARFIMA modeling}

Standard linear forecasting methods have traditionally modeled time series in terms of their short-range behavior. For a given stationary time series $x(t)$, these methods attempt to model a given time series $x(t)$ in terms of autoregressive (AR) and moving average (MA) components. AR components highlight the degree to which a time series $x(t)$ may predicted from the $p$ previous values of itself, and they can be formulated as

$x(t)=\sum_{i=1}^{p} \phi_{i} x(t-i)+\varepsilon(t)$,

where $\phi_{i}$ is a coefficient representing the effect of the $i$ th previous value on the current value of $x(t)$ and $\varepsilon(t)$ is a noise term. MA components highlight the degree to which $x(t)$ may be predicted from Gaussian fluctuations interacting with the $q$ previous values of itself, and they can be formulated as

$x(t)=\sum_{i=1}^{q} \theta_{i} \varepsilon(t-i)+\varepsilon(t)$,

where $\theta_{i}$ is a coefficient representing the effect of the $i$ th previous Gaussian fluctuation. A model with both $p$ AR components and $q$ MA components is called an ARMA $(p, q)$ model (Box \& Jenkins, 1970).

If the time series $x(t)$ is nonstationary, it is said to be have a unit root, meaning that it is the first-order integration of a stationary noise, and AR and MA modeling presupposes stationarity. A common solution is to take the difference $x(t)-x(t-1)$ of the time series $x(t)$, and AR and MA components may be fit to the differences, provided that they are stationary. Hence, the linear model for time series with a unit root is an autoregressive integrated moving average (ARIMA) model that fits AR and MA terms to $x(t)-x(t-1)$, the differences of $x(t)$. In addition to $p$ AR components and $q$ MA components, ARIMA models have a $d$ parameter indicating the number of differencings of $x(t)$ required before $p$ AR and $q$ MA components can be fit to the data (Box \& Jenkins, 1970). These models are denoted as $\operatorname{ARIMA}(p, d, q)$.

ARFIMA modeling is an elaboration of ARIMA modeling. When the parameter $d$ is set to fractional values, the consequent model will exhibit long-range temporal correlations. When $-.5 \leq d<0$, the long-range temporal correlations are negative; when $.5 \geq d>0$, they are positive. It is possible to use ARFIMA modeling to test the presence of long-range temporal correlations (i.e., significantly nonzero $|d| \leq .5$ for the I component) above and beyond short-range temporal correlations (i.e., the $p$ AR and $q$ MA components; Torre et al., 2007; Wagenmakers et al., 2004, 2005). Essentially, this method amounts to fitting nine $\operatorname{ARMA}(p, q)$ models and nine $\operatorname{ARFIMA}(p, d, q)$ models for which $p$ and $q$ can vary among 0 , 1 , and 2 and letting the ARFIMA models estimate the optimized value of $d$. The best-fitting class of models for a given time series $x(t)$ is chosen according to either the AIC or the BIC, defined for the $i$ th model as

$$
\begin{aligned}
& A I C_{i}=-2 \log L_{i}+2 k_{i}, \\
& B I C_{i}=-2 \log L_{i}+k_{i} \log N_{i},
\end{aligned}
$$

where $\log L_{i}, k_{i}$, and $N_{i}$ are the $\log$-likelihood degrees of freedom (i.e., $p+q+1$ for ARMA and $p+q+2$ for ARFIMA), and the length of the time series, respectively, for the $i$ th model (Akaike, 1973; Schwarz, 1978). Each $i$ th model in a set of $m$ models is weighted as $w_{i}(A I C)$ or $w_{i}(B I C)$, where

$$
\begin{aligned}
& \Delta_{i}(A I C)=A I C_{i}-\min A I C, \\
& L_{i}(A I C) \sim \exp \left(-.5^{*} \Delta_{i}(A I C)\right), \\
& w_{i}(A I C)=\frac{L_{i}(A I C)}{\sum_{j=1}^{m} L_{j}(A I C)} .
\end{aligned}
$$

If the sum of weights for ARFIMA models exceeds the sum of weights for ARMA models, and if the highest-weighted ARFIMA model has a significantly nonzero $|d| \leq .5$, the time series can be said to have long-range temporal correlations (Torre et al., 2007). In general, adding parameters to any model should improve its fit. The main question is whether the added parameter - here, $d$ added to the ARMA model in order to produce an ARFIMA - has a significant effect.

\section{Dispersion analysis}

Dispersion analysis examines the change in standard deviation $D$ across sample means of nonoverlapping $n$-length windows of the time series $x(t) . D(n)$ decays according to a power law,

$D(n) \sim n^{-c}$, 
from which follows

$\log D(n) \sim-c \log n$.

The negative slope is used to calculate an estimate of FD,

$F D=1-(-c)$

A time series with long-range temporal correlations will exhibit $1.5>F D \geq 1$ (Aon, Cortassa, \& O'Rourke, 2006; Bassingthwaighte et al., 1994; Van Orden et al., 2003).

Dispersion analysis and DFA assess the same statistical property of fluctuations, but the relationship between the two is not without a superficial ambiguity. Namely, whereas DFA models an $S D$ statistic as a power law function increasing with time scale, dispersion analysis models an $S D$ statistic as an inverse power law decreasing with time scale. Despite the difference of direction, the power laws from each analysis highlight the inexhaustible variability in a hyperdiffusive system. The difference in direction depends on the statistic over which $S D$ is computed. As was noted in the text, the concept of hyperdiffusion addresses the $S D$ of a randomwalk trajectory, not the $S D$ of individual displacements. Whereas the former growth of $S D$ will depend on temporal structure, the latter should not (under CLT). DFA computes the RMS fluctuations in a random-walk trajectory after removing local trends on a variety of time scales. That is, DFA involves computing the average of SDs over progressively longer nonoverlapping stretches of a random-walk trajectory. On the other hand, and in some respects conversely, dispersion analysis involves computing the $S D$ of averages over progressively longer nonoverlapping stretches of individual displacements. Under CLT, averages are summary measures almost guaranteed to compress statistical information and, so, almost guaranteed to become less variable as sample size increases. What distinguishes fractal fluctuations is not that their dispersion curves may decrease but is, instead, that their dispersion curves will decrease much more slowly than those for nonfractal fluctuations. A slow decrease in variability of averages across individual displacements (i.e., evidence of fractality from dispersion analysis) goes hand in hand with a fast increase in average of variability across a random-walk trajectory integrated from individual displacements (i.e., evidence of fractality from DFA). Dispersion analysis and DFA are mutually consistent.

\section{References}

Akaike, H. (1973). Information theory and an extension of the maximum likelihood principle. In B. N. Petrov \& F. Czáki (Eds.), Proceedings of the 2nd International Symposium on Information Theory (pp. 267-281). Budapest: Akademiai Kiadó.
Aks, D. J. (2005). 1/ $f$ dynamic in complex visual search: Evidence for self-organized criticality in human perception. In M. A. Riley \& G. C. Van Orden (Eds.), Tutorials in contemporary nonlinear methods for the behavioral sciences (pp. 326-359). Arlington, VA: National Science Foundation. Retrieved March 1, 2006, from www.nsf.gov/bse/bcs/pac/nmbs/nmbs.jsp

Aks, D. J., Zelinsky, G., \& Sprott, J. C. (2002). Memory across eyemovements: $1 / f$ dynamic in visual search. Nonlinear Dynamics, Psychology, and Life Sciences, 6, 1-25.

Alvarez, G. A., \& Cavanagh, P. (2004). The capacity of visual short-term memory is set both by visual information load and by number of objects. Psychological Science, 15, 106-111.

Anderson, J. R., \& Schooler, L. J. (1991). Reflections of the environment in memory. Psychological Science, 2, 396-408.

Aon, M. A., Cortassa, S., \& O'Rourke, B. (2006). The fundamental organization of cardiac mitochondria as a network of coupled oscillators. Biophysical Journal, 91, 4317-4327.

Baddeley, R., Abbott, L. F., Booth, M. C. A., Sengpiel, F., Freeman, T., Wakeman, E. A., \& Rolls, E. T. (1997). Responses of neurons in primary and inferior temporal visual cortices to natural sources. Proceedings of the Royal Society of London. Series B, 264, 1775-1783.

Banerjee, A., Dolado, J. J., Galbraith, J. W., \& Hendry, D. F. (1993). Cointegration, error correction, and the econometric analysis of non-stationary data. Oxford: Oxford University Press.

Barlow, H. B. (1963). Slippage of contact lenses and other artefacts in relation to fading and regeneration of supposedly stable retinal images. The Quarterly Journal of Experimental Psychology, 15, $36-51$.

Baron-Cohen, S. (1988). Social and pragmatic deficits in autism: Cognitive or affective? Journal of Autism and Developmental Disorders, 18, 379-402.

Bartumeus, F., Catalan, J., Fulco, U. L., Lyra, M. L., \& Viswanathan, G. M. (2002). Optimizing the encounter rate in biological interactions: Lévy versus Brownian strategies. Physical Review Letters, 88, 097901

Bassingthwaighte, J. B., Liebovitch, L. S., \& West, B. J. (1994). Fractal physiology. New York: Oxford University Press.

Billock, V. A., de Guzman, G. C., \& Kelso, J. A. S. (2001). Fractal time and $1 / f$ spectra in dynamic images and human vision. Physica D, 148, 136-146.

Box, G. E. P., \& Jenkins, G. M. (1970). Time series analysis, forecasting and control. San Francisco: Holden-Day.

Brown, G. D. A., Neath, I., \& Chater, N. (2007). A temporal ratio model of memory. Psychological Review, 114, 539576.

Chater, N., \& Brown, G. D. A. (1999). Scale invariance as a unifying psychological principle. Cognition, 69, B17-B24.

Coppola, D., \& Purves, D. (1996). The extraordinary rapid disappearance of entoptic images. Proceedings of the National Academy of Sciences, 93, 8001-8004.

Ditchburn, R. W., \& Ginsborg, B. L. (1952). Vision with a stabilized retinal image. Nature, 170, 36-37.

Dukewich, K. R. (2009). Reconceptualizing inhibition of return as habituation of the orientingresponse. Psychonomic Bulletin \& Review, 16, 238-251.

Fairhall, S. L., Indovina, I., Driver, J., \& Macaluso, E. (2009). The brain network underlying serial visual search: Comparing overt and covert spatial orienting, for activations and for effective connectivity. Cerebral Cortex, 19, 2946-2958.

Friston, K. (2009). The free-energy principle: A rough guide to the brain? Trends in Cognitive Sciences, 13, 293-301.

Granger, C. W. J., \& Joyeux, R. (1980). An introduction to long-memory models and fractional differencing. Journal of Time Series Analysis, $1,15-29$. 
Happé, F. (1999). Autism: Cognitive deficit or cognitive style? Trends in Cognitive Sciences, 3, 216-222.

Heinrich, J. (2003). Pitfalls of goodness-of-fit from likelihood. In L. Lyons, R. P. Mount, \& R. Reitmeyer (Eds.), Proceedings of the conference on statistical problems in particle physics, astrophysics, and cosmology (pp. 52-55). Stanford: SLAC.

Helmholtz, H. (1962). Treatise on physiological optics (J. P. C. Southall, Trans.). New York: Dover. (Original work published in 1866).

Hill, E. L., \& Frith, U. (2003). Understanding autism: Insights from mind and brain. Philosophical Transactions of the Royal Society of London, B, 358, 281-289.

Holden, J. G. (2005). Gauging the fractal dimension of cognitive performance. In M. A. Riley \& G. C. Van Orden (Eds.), Tutorials in contemporary nonlinear methods for the behavioral sciences (pp. 353-400). Retrieved February 20, 2005, from http://www. nsf.gov/sbe/bcs/pac/nmbs/nmbs.jsp

Joseph, R. M., Keehn, B., Connolly, C., Wolfe, J. M., \& Horowitz, T. S. (2009). Why is visual search superior in autism spectrum disorder? Developmental Science, 12, 1083-1096.

Kanner, L. (1943). Autistic disturbance of affective contact. The Nervous Child, 2, 217-250.

Kello, C. T., Beltz, B. C., Holden, J. G., \& Van Orden, G. C. (2007). The emergent coordination of cognitive function. Journal of Experimental Psychology: General, 136, 551-558.

Kiefer, A. W., Riley, M. A., Shockley, K., Villard, S., \& Van Orden, G. C. (2009). Walking changes the dynamics of cognitive estimates of time intervals. Journal of Experimental Psychology: Human Perception and Performance, 35, 1532-1541.

Klein, R. M. (2000). Inhibition of return. Trends in Cognitive Sciences, 4, 138-147.

Kugler, P. N., \& Turvey, M. T. (1987). Information, natural law, and the self-assembly of rhythmic movement. Hillsdale, NJ: Erlbaum.

Lowen, S. B., Ozaki, T., Kaplan, E., Saleh, B. E. A., \& Teich, M. C. (2001). Fractal features of dark, maintained, and driven neural discharges in the cat visual system. Methods, 24, 377-394.

Lyons, L. (2008). Open statistical issues in particle physics. Annals of Applied Statistics, 3, 887-915.

Maraun, D., Rust, H., \& Timmer, J. (2004). Tempting long-memory —on the interpretation of DFA results. Nonlinear Processes in Geophysics, $11,495-503$.

Mavritsaki, E., Allen, H. A., \& Humphreys, G. W. (2010). Decomposing the neural mechanisms of visual search through model-based analysis of fMRI: Top-down excitation, active ignoring and the use of saliency by the right TPJ. Neuroimage. doi:10.1016/j. neuroimage.2010.03.044

Miller, J., \& Festinger, L. (1977). Impact of oculomotor retraining on the visual perception of curvature. Journal of Experimental Psychology: Human Perception and Performance, 3, 187-200.

Mirman, D., Dixon, J. A., \& Magnuson, J. S. (2008). Statistical and computations models of the visual world paradigm: Growth curves and individual differences. Journal of Memory and Language, 59, 475-494.

O'Riordan, M. A. (2004). Superior visual search in adults with autism. Autism, 8, 229-248.

O'Riordan, M., Plaisted, K., Driver, J., \& Baron-Cohen, S. (2001). Superior visual search in autism. Journal of Experimental Psychology: Human Perception and Performance, 27, 719-730.

Oswiecimka, P., Kwapien, J., \& Drozdz, S. (2006). Wavelet versus detrended fluctuation analysis of multifractal structures. Physical Review E, 74, 016103.

Ozonoff, S., \& Strayer, D. L. (2001). Further evidence of intact working memory in autism. Journal of Autism and Developmental Disorders, 31, 257-263.

Peng, C.-K., Buldyrev, S. V., Havlin, S., Simons, M., Stanley, H. E., \& Goldberger, A. L. (1994). Mosaic organization of DNA nucleotides. Physical Review E, 49, 1685-1689.
Pizlo, Z. (2008). 3D shape: Its unique place in visual perception. Cambridge, MA: MIT Press.

Posner, M. I., \& Cohen, Y. (1984). Components of visual orienting. In H. Bouma \& D. G. Bouwhuis (Eds.), Attention \& performance $X$ : Control of language processes (pp. 531-556). Hillsdale, NJ: Erlbaum.

Posner, M. I., Rafal, R. D., Choate, L. S., \& Vaughan, J. (1985). Inhibition of return: Neural basis and function. Cognitive Neuroscience, 2, 211-228.

Pritchard, R. M., Heron, W., \& Hebb, D. O. (1960). Visual perception approached by the method of stabilized images. Canadian Journal of Psychology, 14, 67-77.

Ratcliff, R. (1978). A theory of memory retrieval. Psychological Review, 85, 59-108.

Reynolds, A. M. (2010). Bridging the gulf between correlated random walks and Lévy walks: Autocorrelation as a source of Lévy walk movement patterns. Journal of the Royal Society, Interface, 7, $1753-1758$.

Rhodes, T., \& Turvey, M. T. (2007). Human memory retrieval as Lévy foraging. Physica A, 385, 255-260.

Riggs, L. A., \& Ratliff, F. (1952). The effects of counteracting the normal movements of the eye. Journal of the Optical Society of America, 42, 872-873.

Riggs, L. A., Ratliff, F., Cornsweet, J. C., \& Cornsweet, T. N. (1953). The disappearance of steadily fixated visual test objects. Journal of the Optical Society of America, 43, 495-501.

Robertson, S. S., \& Johnson, S. L. (2008). Embodied infant attention. Developmental Science, 12, 297-304.

Rutter, M. (1983). Cognitive deficits in the pathogenesis of autism. Journal of Child Psychology and Psychiatry, 24, 513 531.

Scafetta, N., \& Grigolini, P. (2002). Scaling detection in time series: Diffusion entropy analysis. Physical Review E, 66, 036130.

Schwarz, G. E. (1978). Estimating the dimension of a model. Annals of Statistics, 6, 461-464.

Shlesinger, M. F., Zaslavsky, G. M., \& Klafter, J. (1993). Strange kinetics. Nature, 363, 31-37.

Shore, D. I., \& Klein, R. M. (2000). On the manifestations of memory in visual search. Spatial Vision, 14, 59-76.

Singer, J. D., \& Willett, J. B. (2003). Applied longitudinal data analysis: Modeling change and event occurrence. New York: Oxford University Press.

Snyder, J. J., \& Kingstone, A. (2007). Inhibition of return at multiple locations and its impact on visual search. Visual Cognition, 15, $238-256$

Stephen, D. G., Arzamarski, R., \& Michaels, C. F. (2010). The role of fractality in perceptual learning: Exploration in dynamic touch. Journal of Experimental Psychology: Human Perception and Performance, 36, 1161-1173.

Stephen, D. G., Boncoddo, R. A., Magnuson, J. S., \& Dixon, J. A. (2009). The dynamics of insight: Mathematical discovery as a phase transition. Memory \& Cognition, 37, 1132-1149.

Stephen, D. G., \& Mirman, D. (2010). Interactions dominate the dynamics of cognition. Cognition, 115, 154-165.

Stephen, D. G., Mirman, D., Magnuson, J. S., \& Dixon, J. A. (2009). Lévy-like diffusion in eye movements during spoken-language comprehension. Physical Review E, 79, 056114.

Tanenhaus, M. K., Spivey-Knowlton, M. J., Eberhard, K. M., \& Sedivy, J. C. (1995). Integration of visual and linguistic information in spoken language comprehension. Science, 268, $1632-1634$.

Thelen, E., \& Smith, L. B. (1994). A dynamic systems approach to the development of cognition and action. Cambridge, MA: MIT Press.

Torre, K., Delignières, D., \& Lemoine, L. (2007). Detection of long-range dependence and estimation of fractal exponents through ARFIMA 
modeling. The British Journal of Mathematical and Statistical Psychology, 60, 85-106.

Treisman, A. M. (1996). The binding problem. Current Opinions in Neurobiology, 6, 171-178.

Treisman, A. M., \& Gelade, G. (1980). A feature-integration theory of attention. Cognitive Psychology, 12, 97-136.

Van Orden, G. C., Holden, J. G., \& Turvey, M. T. (2003). Selforganization of cognitive performance. Journal of Experimental Psychology: General, 132, 331-350.

Wagenmakers, E.-J., Farrell, S., \& Ratcliff, R. (2004). Estimation and interpretation of $1 / f^{\alpha}$ noise in human cognition. Psychonomic Bulletin \& Review, 11, 579-615.

Wagenmakers, E.-J., Farrell, S., \& Ratcliff, R. (2005). Human cognition and a pile of sand: A discussion on serial correlations and self-organized criticality. Journal of Experimental Psychology: General, 134, 108-116.

Wagenmakers, E.-J., Ratcliff, R., Gomez, P., \& McKoon, G. (2008). A diffusion model account of criterion shifts in the lexical decision task. Journal of Memory and Language, 58, 140-159.

Werner, G. (2010). Fractals in the nervous system: Conceptual implications for theoretical neuroscience. Frontiers in Physiology, $1,1-20$.

West, B. J. (2006). Where medicine went wrong: Rediscovering the path to complexity. Singapore: World Scientific.

Wolfe, J. M., Cave, K. R., \& Franzel, S. L. (1989). Guided search: An alternative to the feature integration model for visual search. Journal of Experimental Psychology: Human Perception and Performance, 15, 419-433. 